Bull. Austral. Math. Soc.

$35 \mathrm{~A} 15,35 \mathrm{~B} 30,35 \mathrm{~B} 35,35 \mathrm{G} 20,35 \mathrm{~J} 45,49 \mathrm{~N} 15$

VoL. $76(2007)$ [453-470]

\title{
ON EXISTENCE AND STABILITY OF SOLUTIONS TO ELLIPTIC SYSTEMS WITH GENERALISED GROWTH
}

\section{Marek Galewski and Marek Peócienniczak}

\begin{abstract}
We are concerned with existence and stability of solutions for system of equations with generalised $p(x)$ and $m(x)$-Laplace operators and where the nonlinearity satisfies some local growth conditions. We provide a variational approach that is based on investigation of the primal and the dual action functionals. As a consequence we consider the dependence of the the system on functional parameters.
\end{abstract}

\section{INTRODUCTION}

In this paper we consider existence and stability of solutions to the following family of systems of Dirichlet problems with generalised $p(x), m(x)$-Laplacian operators for $k=0,1,2, \ldots$

$$
\begin{aligned}
&-\operatorname{div}\left(a(x)|\nabla u(x)|^{p(x)-2} \nabla u(x)\right)=F_{u}^{k}(x, u(x), v(x)), \\
&-\operatorname{div}\left(b(x)|\nabla v(x)|^{m(x)-2} \nabla v(x)\right)=F_{v}^{k}(x, u(x), v(x)), \\
&\left.u(x)\right|_{\partial \Omega}=0, u \in W_{0}^{1, p(x)}(\Omega),\left.\quad v(x)\right|_{\partial \Omega}=0, v \in W_{0}^{1, m(x)}(\Omega)
\end{aligned}
$$

where $\Omega \subset R^{N}$ is a bounded region with Lipschitz boundary, $p, q, m, n \in C(\bar{\Omega}), 1 / p(x)$ $+1 / q(x)=1,1 / m(x)+1 / n(x)=1$ for $x \in \Omega ; W_{0}^{1, p(x)}(\Omega), W_{0}^{1, m(x)}(\Omega)$ denote the generalised Orlicz-Sobolev spaces, see $[3,5] ; a, b \in C(\bar{\Omega})$ with $a(x) \geqslant a_{0}>0$, $b(x) \geqslant b_{0}>0$ on $\bar{\Omega}$ for $k=0,1,2, \ldots$. Let $p^{-}=\inf _{x \in \Omega} p(x)>N, m^{-}=\inf _{x \in \Omega} m(x)>N$.

We shall show - upon some conditions - that for all $k=1,2, \ldots$ there exists a solution $\left(u_{k}, v_{k}\right)$ to (1.2) and later that from the sequence $\left(u_{k}, v_{k}\right)$ one can choose a subsequence $\left(u_{k_{i}}, v_{k_{i}}\right)$ such that $u_{k_{i}} \rightarrow \bar{u}$ weakly in $W^{1, p(x)}(\Omega), v_{k_{i}} \rightarrow \bar{v}$ weakly in $W^{1, m(x)}(\Omega)$ and

$$
\begin{aligned}
&-\operatorname{div}\left(a(x)|\nabla \bar{u}(x)|^{p(x)-2} \nabla \bar{u}(x)\right)=F_{u}^{0}(x, \bar{u}(x), \bar{v}(x)), \\
&-\operatorname{div}\left(b(x)|\nabla \bar{v}(x)|^{m(x)-2} \nabla \bar{v}(x)\right)=F_{v}^{0}(x, \bar{u}(x), \bar{v}(x)), \\
&\left.\bar{u}(x)\right|_{\partial \Omega}=0,\left.\quad \bar{v}(x)\right|_{\partial \Omega}=0 .
\end{aligned}
$$

Received 16th April, 2007

The research of the second author is supported by the European Social Fund and Budget of State implemented under the Integrated Regional Operational Programme. Project: GRRI-D.

Copyright Clearance Centre, Inc. Serial-fee code: 0004-9727/07 \$A2.00+0.00. 
Such a property we shall call the stability of the system. Some general framework for studying stability of solutions to variational problems in sublinear case can be found in $[10,12]$ and $[13]$ but our method provides suitable results for the family of systems of Dirichlet problems with generalised $p(x), m(x)$-Laplacian operators.

In order to obtain the solution to (1.2) we minimise $J_{k}$ on a set $X_{k} \subset W^{1, p(x)}(\Omega)$ $\times W^{1, m(x)}(\Omega)$ which has the following property: for all $(u, v) \in X_{k}$, the relation

$$
\begin{aligned}
& -\operatorname{div}\left(a(x)|\nabla \tilde{u}(x)|^{p(x)-2} \nabla \tilde{u}(x)\right)=F_{u}^{k}(x, u(x), v(x)), \\
& -\operatorname{div}\left(b(x)|\nabla \tilde{v}(x)|^{m(x)-2} \nabla \tilde{v}(x)\right)=F_{v}^{k}(x, u(x), v(x)) \\
& \left.\tilde{u}(x)\right|_{\partial \Omega}=0, \tilde{u} \in W_{0}^{1, p(x)}(\Omega),\left.\quad \tilde{v}(x)\right|_{\partial \Omega}=0, \tilde{v} \in W_{0}^{1, m(x)}(\Omega)
\end{aligned}
$$

implies $(\tilde{u}, \tilde{v}) \in X_{k}$.

First we show, with the aid growth conditions F1, F2, F3 (see Section 2), that the action functional

$$
J_{k}(u, v)=\int_{\Omega} \frac{a(x)}{p(x)}|\nabla u(x)|^{p(x)} d x+\int_{\Omega} \frac{b(x)}{m(x)}|\nabla v(x)|^{m(x)} d x-\int_{\Omega} F^{k}(x, u(x), v(x)) d x
$$

is bounded from below and achieves its minimum $\left(\bar{u}_{k}, \bar{v}_{k}\right)$ on $X_{k}$. Since $X_{k}$ is not dense in $W^{1, p(x)}(\Omega) \times W^{1, m(x)}(\Omega)$ we may not apply the Euler-Lagrange equation. Our assumptions also do not allow us to use either the mountain pass geometry or the topological approach. In order to show that $\left(\bar{u}_{k}, \bar{v}_{k}\right)$ is indeed a solution we construct a dual functional $J_{k}^{D}$ : $W^{1} \times W^{2} \rightarrow R$

$$
\begin{aligned}
J_{k}^{D}(w, z)= & \int_{\Omega}\left(F^{k}\right)^{*}(x,-\operatorname{div} w(x),-\operatorname{div} z(x)) d x \\
& -\int_{\Omega} \frac{1}{(a(x))^{q(x) / p(x)}} \frac{1}{q(x)}|w(x)|^{q(x)} d x-\int_{\Omega} \frac{1}{(b(x))^{n(x) / m(x)}} \frac{1}{n(x)}|v(x)|^{n(x)} d x
\end{aligned}
$$

where

$$
\begin{aligned}
& W^{1}=\left\{w \in L^{q(x)}(\Omega) \mid \operatorname{div} w \in L^{g(x)}(\Omega)\right\} \\
& W^{2}=\left\{z \in L^{n(x)}(\Omega) \mid \operatorname{div} z \in L^{n(x)}(\Omega)\right\}
\end{aligned}
$$

and investigate relations between $J$ and $J_{k}^{D}$. We relate critical values on $X_{k}$ and $X_{k}^{d}$ (on which $J_{k}^{D}$ is considered) and later we relate the relevant critical points. These relations provide the existence of solutions. Construction of $X_{k}$ and some convergence of $F^{k}$ will further allow us to obtain stability. Here $\left(F^{k}\right)^{*}$ denotes the Fenchel-Young conjugate of $F^{k}$, see [2], that is,

$$
\left(F^{k}\right)^{*}\left(x, w_{1}, w_{2}\right)=\sup _{z \in R^{2}}\left(\langle w, z\rangle-F^{k}\left(x, z_{1}, z_{2}\right)\right)
$$

where $z=\left(z_{1}, z_{2}\right)$ and $w=\left(w_{1}, w_{2}\right)$. The only work - known to the authors - that concerns elliptic system with generalised growth is [9]. Following [4] the authors of [9] 
apply first a direct method of the calculus of variations and later a mountain pass geometry. Since problems with generalised growth conditions are applied in elastic mechanics and electrorheological fluid dynamics (see $[11,14]$ and references therein), we believe that our results may contribute to that research. Concerning some ideas on stability in variational problems we may mention [6].

\section{ASSUMPTIONS AND AUXILIARY RESULTS}

In what follows by $C_{S}^{1}, C_{S}^{2}$ we denote the best Sobolev constants

$$
\begin{gathered}
\|u\|_{p(x)} \leqslant C_{S}^{1}\|\nabla u\|_{p(x)} \text { for all } u \in W_{0}^{1, p(x)}(\Omega), \\
\|u\|_{m(x)} \leqslant C_{S}^{2}\|\nabla u\|_{m(x)} \text { for all } u \in W_{0}^{1, m(x)}(\Omega) .
\end{gathered}
$$

Since $W_{0}^{1, p(x)}(\Omega)$ is continuously embedded into $W_{0}^{1, p^{-}}(\Omega)$, as well as $W_{0}^{1, m(x)}(\Omega)$ into $W_{0}^{1, m^{-}}(\Omega),[3]$, we denote by $C_{1}^{m}, C_{1}^{p}$ the following constants

$$
\begin{gathered}
\|\nabla u\|_{p^{-}} \leqslant C_{1}^{p}\|\nabla u\|_{p(x)}, \\
\|\nabla v\|_{m^{-}} \leqslant C_{1}^{m}\|\nabla v\|_{m(x)} .
\end{gathered}
$$

Since $p^{-}>N$ and $m^{-}>N$ by Sobolev Imbedding Theorem [1] we get

$$
\begin{aligned}
& \max _{x \in \Omega}|u(x)| \leqslant C_{2}^{p}\|\nabla u\|_{p^{-}} \text {for all } u \in W_{0}^{1, p^{-}}(\Omega), \\
& \max _{x \in \Omega}|v(x)| \leqslant C_{2}^{m}\|\nabla v\|_{m^{-}} \text {for all } v \in W_{0}^{1, m^{-}}(\Omega) .
\end{aligned}
$$

Therefore by (2.2) and (2.3) for all $u \in W_{0}^{1, p(x)}(\Omega), v \in W_{0}^{1, m(x)}(\Omega)$ we get

$$
\begin{aligned}
& \max _{x \in \Omega}|u(x)| \leqslant C_{2}^{p}\|\nabla u\|_{p^{-}} \leqslant C_{1}^{p} C_{2}^{p}\|\nabla u\|_{p(x)}, \\
& \max _{x \in \Omega}|v(x)| \leqslant C_{2}^{m}\|\nabla v\|_{m^{-}} \leqslant C_{1}^{m} C_{2}^{m}\|\nabla v\|_{m(x)} .
\end{aligned}
$$

Let us consider two nondecreasing sequences of positive numbers, bounded away from $0,\left\{d_{k}\right\}_{k=1}^{\infty},\left\{c_{k}\right\}_{k=1}^{\infty}$. We assume that

F1: $\|1\|_{q(x)} \leqslant\left(1 / p^{-}+1 / q^{-}\right)^{-1},\|1\|_{n(x)} \leqslant\left(1 / m^{-}+1 / n^{-}\right)^{-1}$ and for $k=0,1,2, \ldots$

$$
\begin{gathered}
C_{S}^{1} C_{1}^{p} C_{2}^{p} \text { ess sup } \max _{x \in \Omega} \max _{u \in\left[-d_{k}, d_{k}\right]}\left|F_{v \in\left[-c_{k}, c_{k}\right.}\right| F_{u}^{k}(x, u, v) \mid \leqslant a_{0} d_{k}, \\
C_{S}^{2} C_{1}^{m} C_{2}^{m} \text { ess sup } \max _{x \in \Omega} \max _{u \in\left[-d_{k}, d_{k}\right]}\left|F_{v \in\left[-c_{k}, c_{k}\right]}^{k}(x, u, v)\right| \leqslant b_{0} c_{k} .
\end{gathered}
$$

F2: $\quad F^{k}, F_{u}^{k}, F_{v}^{k}: \Omega \times\left[-d_{0}, d_{0}\right] \times\left[-c_{0}, c_{0}\right] \rightarrow R$ are Caratheodory functions for all $k=0,1,2, \ldots, F^{k}$ is convex in the last two variables on $\left[-d_{k}, d_{k}\right] \times\left[-c_{k}, c_{k}\right]$ for all $k=0,1,2, \ldots$ and almost all $x \in \Omega$.

We may define $F^{k}$ on $\Omega \times\left(R \backslash\left[-d_{0}, d_{0}\right]\right) \times\left(R \backslash\left[-c_{0}, c_{0}\right]\right)$ by putting $F^{k}=+\infty$. Now $F^{k}$ is convex and lower semicontinuous. 
F3 $F_{u}^{k}(x, 0,0) \neq 0, F_{v}^{k}(x, 0,0) \neq 0$ for almost all $x \in \Omega$, functions $x \mapsto$ $F^{k}(x, 0,0)$ and $x \mapsto\left(F^{k}\right)^{*}(x, 0,0)$ are integrable on $\Omega$ where $\left(F^{k}\right)^{*}$ is defined by (1.3).

We put

$$
\begin{array}{r}
X_{k}=\left\{(u, v) \in W_{0}^{1, p(x)}(\Omega) \times W_{0}^{1, m(x)}(\Omega),\|\nabla u\|_{p(x)} \leqslant \frac{d_{k}}{C_{1}^{p} C_{2}^{p}},\|\nabla v\|_{m(x)} \leqslant \frac{c_{k}}{C_{1}^{m} C_{2}^{m}},\right. \\
\left.|u(x)| \leqslant d_{k},|v(x)| \leqslant c_{k}\right\} .
\end{array}
$$

Reasoning exactly as in [8] we show that $X_{k}$ has indeed property (1.3). The dual functional $J_{k}^{D}$ will be considered on a set $X_{k}^{d}$ which is a set of these $(w, z) \in W^{1} \times W^{2}$ for which there exists a $(u, v) \in X_{k}$ such that

$$
-\operatorname{div} w(x)=F_{u}^{k}(x, u(x), v(x))
$$

and

$$
\begin{gathered}
a(x)|\nabla \widetilde{u}(x)|^{p(x)-2} \nabla \widetilde{u}(x)=w(x), \\
-\operatorname{div} z(x)=F_{v}^{k}(x, u(x), v(x))
\end{gathered}
$$

and

$$
b(x)|\nabla \widetilde{v}(x)|^{m(x)-2} \nabla \widetilde{v}(x)=z(x),
$$

where $(\widetilde{u}, \widetilde{v})$ corresponds to $(u, v)$ in (1.3).

$J_{k}$ and $J_{k}^{D}$ are well defined on $X_{k}$ and $X_{k}^{d}$ due to the following.

LEMMA 2.1. For any $k=0,1,2, \ldots$, there exist constants $\gamma_{k}, \eta_{k}>0$ such that

$$
\left|\int_{\Omega} F^{k}(x, u(x), v(x)) d x\right| \leqslant \gamma_{k}
$$

for all $(u, v) \in X_{k}$ and

$$
\left|\int_{\Omega}\left(F^{k}\right)^{*}(x,-\operatorname{div} w(x),-\operatorname{div} z(x)) d x\right| \leqslant \eta_{k}
$$

for all $(w, z) \in X_{k}^{d}$.

PROOF: Relation (2.10) follows by convexity of $F^{k}, F 1, F 3$ and the estimates

$$
\left|F^{k}(x, u(x), v(x))\right| \leqslant\left|F^{k}(x, 0,0)\right|+\sup _{x \in \Omega}\left\{\left|F_{u}^{k}(x, 0,0)\right||u(x)|\right\} .
$$

By (2.6), (2.8), (2.10) and the definition of $X_{k}^{d}$ we get that

$$
\begin{aligned}
\int_{\Omega}\left(F^{k}\right)^{*}(x,- & \operatorname{div} w(x),-\operatorname{div} z(x)) d x \\
& =-\int_{\Omega} F^{k}(x, u(x), v(x)) d x+\int_{\Omega}(u(x), v(x))(-\operatorname{div} w(x),-\operatorname{div} z(x)) d x
\end{aligned}
$$

is finite. Thus relation (2.11) follows. 


\section{EXISTENCE OF SOLUTIONS}

THEOREM 3.1. Assume F1, F2, F3. For all $k=0,1,2, \ldots$ there exists $\left(u_{k}, v_{k}, w_{k}, z_{k}\right) \in X_{k} \times X_{k}^{d}$ such that

$$
\begin{gathered}
-\operatorname{div} w_{k}(x)=F_{u}^{k}\left(x, u_{k}(x), v_{k}(x)\right),-\operatorname{div} z_{k}(x)=F_{v}^{k}\left(x, u_{k}(x), v_{k}(x)\right), \\
a(x)\left|\nabla u_{k}(x)\right|^{p(x)-2} \nabla u_{k}(x)=w_{k}(x), b(x)\left|\nabla v_{k}(x)\right|^{m(x)-2} \nabla v_{k}(x)=z_{k}(x)
\end{gathered}
$$

Moreover

$$
\inf _{(w, z) \in X_{k}^{d}} J_{k}^{D}(w, z)=J_{k}^{D}\left(w_{k}, z_{k}\right)=J_{k}\left(u_{k}, v_{k}\right)=\inf _{(u, v) \in X_{k}} J_{k}(u, v) .
$$

Proof: We fix $k=0,1,2 \ldots$ We observe that by Lemma 2.1

$$
\begin{aligned}
J_{k}(u, v)=\int_{\Omega} \frac{a(x)}{p(x)}|\nabla u(x)|^{p(x)} d x & +\int_{\Omega} \frac{b(x)}{m(x)}|\nabla v(x)|^{m(x)} d x \\
& -\int_{\Omega} F^{k}(x, u(x), v(x)) d x \geqslant-\gamma_{k} .
\end{aligned}
$$

Therefore $\inf _{(u, v) \in X_{k}} J_{k}(u, v)$ is finite. By the properties of $X_{k}$ there exists a minimising sequence $\left\{\left(u_{k}^{n}, v_{k}^{n}\right)\right\}_{n=1}^{\infty}$ for functional $J_{k}$ on $X_{k}$ and this sequence may be assumed to be weakly convergent in $W_{0}^{1, p(x)}(\Omega) \times W_{0}^{1, m(x)}(\Omega)$ and therefore, up to a subsequence, strongly in $L^{p(x)}(\Omega) \times L^{m(x)}(\Omega)$. Thus it contains a subsequence convergent almost everywhere, still denoted by $\left\{\left(u_{k}^{n}, v_{k}^{n}\right)\right\}_{n=1}^{\infty}$ and its limit is denoted by $\left(u_{k}, v_{k}\right)$. We see that

$$
\left\|\nabla u_{k}^{n}\right\|_{L^{p(x)}(\Omega)} \leqslant \frac{d_{k}}{C_{1}^{p} C_{2}^{p}}
$$

for all $n$ and

$$
\liminf _{n \rightarrow \infty}\left\|\nabla u_{k}^{n}\right\|_{L^{p(x)}(\Omega)} \geqslant\left\|\nabla u_{k}\right\|_{L^{p(x)}(\Omega)} .
$$

Therefore $\left\|\nabla u_{k}\right\|_{L^{p(x)}(\Omega)} \leqslant\left(d_{k}\right) /\left(C_{1}^{p} C_{2}^{p}\right)$. By definition of sequence $\left\{u_{k}^{n}\right\}_{n=1}^{\infty}$ we also get $\left|u_{k}^{n}(x)\right| \leqslant d_{k}$. Since $\left\{u_{k}^{n}\right\}_{n=1}^{\infty}$ is convergent almost everywhere, we get $\left|u_{k}(x)\right| \leqslant d_{k}$. The same holds for $\left\{v_{k}^{n}\right\}_{n=1}^{\infty}$. So $\left(u_{k}, v_{k}\right) \in X_{k}$ and we get $\liminf _{n \rightarrow \infty} J_{k}\left(u_{k}^{n}, v_{k}^{n}\right) \geqslant J_{k}\left(u_{k}, v_{k}\right)$ since

$$
\lim _{n \rightarrow \infty} \int_{\Omega} F_{u}^{k}\left(x, u_{k}^{n}(x), v_{k}^{n}(x)\right) d x=\int_{\Omega} F_{u}^{k}\left(x, u_{k}(x), v_{k}(x)\right) d x .
$$

Thus $J_{k}$ is weakly lower semicontinuous on $X_{k}$ and since $X_{k}$ is weakly compact we see that $J_{k}\left(u_{k}, v_{k}\right)=\inf _{(u, v) \in X_{k}} J_{k}(u, v)$.

We show that

$$
\inf _{(w, z) \in X_{k}^{d}} J_{k}^{D}(w, z)=\inf _{(u, v) \in X_{k}} J_{k}(u, v)
$$


We consider a functional $J_{k}^{\#}: X_{k} \times X_{k}^{d} \rightarrow R$ given by the formula

$$
\begin{aligned}
J_{k}^{\#}(u, v, w, z)= & \int_{\Omega}\left(F^{k}\right)^{*}(x,-\operatorname{div} w(x),-\operatorname{div} z(x)) d x+\int_{\Omega} \frac{a(x)}{p(x)}|\nabla u(x)|^{p(x)} d x \\
& -\int_{\Omega} \nabla u(x) w(x) d x+\int_{\Omega} \frac{b(x)}{m(x)}|\nabla v(x)|^{m(x)} d x-\int_{\Omega} \nabla v(x) z(x) d x .
\end{aligned}
$$

We observe that for any $(u, v) \in X_{k}$

$$
\inf _{(w, z) \in X_{k}^{d}} J_{k}^{\#}(u, v, w, z)=J_{k}(u, v)
$$

and for any $(w, z) \in X_{k}^{d}$

$$
\inf _{(u, v) \in X_{k}} J_{k}^{\#}(u, v, w, z)=J_{k}^{D}(w, z)
$$

To show (3.5) we fix $(u, v) \in X_{k}$ and obtain by Fenchel-Young inequality

$$
\begin{aligned}
& \sup _{(w, z) \in X_{k}^{d}} \int_{\Omega}[(u(x), v(x))(-\operatorname{div} w(x),-\operatorname{div} z(x)) \\
&\left.-\left(F^{k}\right)^{*}(x,-\operatorname{div} w(x),-\operatorname{div} z(x))\right] d x \leqslant \int_{\Omega} F^{k}(x, u(x), v(x)) d x .
\end{aligned}
$$

By definition of $X_{k}^{d}$ there exists $(\widehat{w}, \widehat{z}) \in X_{k}^{d}$ satisfying

$$
\begin{aligned}
-\operatorname{div} \widehat{w}(x) & =F_{u}^{k}(x, u(x), v(x)), \\
-\operatorname{div} \widehat{z}(x) & =F_{v}^{k}(x, u(x), v(x)),
\end{aligned}
$$

which provides

$$
\begin{aligned}
\int_{\Omega}(u(x), v(x)) & (-\operatorname{div} \widehat{w}(x),-\operatorname{div} \widehat{z}(x)) d x \\
- & \int_{\Omega}\left(F^{k}\right)^{*}(x,-\operatorname{div} \widehat{w}(x),-\operatorname{div} \widehat{z}(x)) d x=\int_{\Omega} F^{k}(x, u(x), v(x)) d x .
\end{aligned}
$$

Therefore equality holds in (3.7). This and integration by parts provides

$$
\begin{aligned}
& \inf _{(w, z) \in X_{k}^{d}} J_{k}^{\#}(u, v, w, z) \\
& =-\sup _{(w, z) \in X_{k}^{d}}\left[-\int_{\Omega}\left(F^{k}\right)^{*}(x,-\operatorname{div} w(x),-\operatorname{div} z(x)) d x\right. \\
& \left.\quad+\int_{\Omega} \nabla u(x) w(x) d x+\int_{\Omega} \nabla v(x) z(x) d x\right] \\
& \quad+\int_{\Omega} \frac{a(x)}{p(x)}|\nabla u(x)|^{p(x)} d x+\int_{\Omega} \frac{b(x)}{m(x)}|\nabla v(x)|^{m(x)} d x
\end{aligned}
$$




$$
\begin{aligned}
= & -\sup _{(w, z) \in X_{k}^{d}}\left[-\int_{\Omega}\left(F^{k}\right)^{*}(x,-\operatorname{div} w(x),-\operatorname{div} z(x)) d x\right. \\
& \left.+\int_{\Omega}(u(x), v(x))(-\operatorname{div} w(x),-\operatorname{div} z(x))\right] \\
& +\int_{\Omega} \frac{a(x)}{p(x)}|\nabla u(x)|^{p(x)} d x+\int_{\Omega} \frac{b(x)}{m(x)}|\nabla v(x)|^{m(x)} d x \\
= & -\int_{\Omega} F^{k}(x, u(x), v(x)) d x+\int_{\Omega} \frac{a(x)}{p(x)}|\nabla u(x)|^{p(x)} d x+\int_{\Omega} \frac{b(x)}{m(x)}|\nabla v(x)|^{m(x)} d x=J_{k}(u, v)
\end{aligned}
$$

so (3.5) follows.

To show (3.6) we fix $(w, z) \in X_{k}^{d}$. We obtain by the Fenchel-Young inequalities

$$
\begin{aligned}
\sup _{(u, v) \in X_{k}} & \left\{\int_{\Omega} w(x) \nabla u(x) d x-\int_{\Omega} \frac{a(x)}{p(x)}|\nabla u(x)|^{p(x)} d x\right. \\
& \left.\quad+\int_{\Omega} \nabla v(x) z(x) d x-\int_{\Omega} \frac{b(x)}{m(x)}|\nabla v(x)|^{m(x)} d x\right\} \\
\leqslant & \int_{\Omega} \frac{1}{(a(x))^{q(x) / p(x)}} \frac{1}{q(x)}|w(x)|^{q(x)} d x+\int_{\Omega} \frac{1}{(b(x))^{n(x) / m(x)}} \frac{1}{n(x)}|v(x)|^{n(x)} d x
\end{aligned}
$$

For a given $(w, z) \in X_{k}^{d}$ there exists $(\tilde{u}, \tilde{v}) \in X_{k}$ such that

$$
a(x)|\nabla \widetilde{u}(x)|^{p(x)-2} \nabla \widetilde{u}(x)=w(x), b(x)|\nabla \widetilde{v}(x)|^{m(x)-2} \nabla \widetilde{v}(x)=z(x) .
$$

Thus we get

$$
\begin{gathered}
\int_{\Omega} w(x) \nabla \widetilde{u}(x) d x-\int_{\Omega} \frac{a(x)}{p(x)}|\nabla \widetilde{u}(x)|^{p(x)} d x+\int_{\Omega} z(x) \nabla \widetilde{v}(x) d x-\int_{\Omega} \frac{b(x)}{m(x)}|\nabla \widetilde{v}(x)|^{m(x)} d x \\
=\int_{\Omega} \frac{1}{(a(x))^{q(x) / p(x)}} \frac{1}{q(x)}|w(x)|^{q(x)} d x+\int_{\Omega} \frac{1}{(b(x))^{n(x) / m(x)}} \frac{1}{n(x)}|v(x)|^{n(x)} d x .
\end{gathered}
$$

Thus equality holds in (3.10) and relation (3.6) follows.

By (3.5) and (3.6) we obtain

$\inf _{(u, v) \in X_{k}} J_{k}(u, v)=\inf _{(u, v) \in X_{k}} \inf _{(w, z) \in X_{k}^{d}} J_{k}^{\#}(u, v, w, z)=\inf _{(w, z) \in X_{k}^{d}(u, v) \in X_{k}} J_{k}^{\#}(u, v, w, z)=\inf _{(w, z) \in X_{k}^{d}} J_{k}^{D}(w, z)$ and (3.4) follows.

Since $\left(u_{k}, v_{k}\right) \in X_{k}$ we may take $\left(w_{k}, z_{k}\right) \in X_{k}^{d}$ such that (3.1) hold. By the FenchelYoung inequalities

$$
\int_{\Omega} \frac{a(x)}{p(x)}\left|\nabla u_{k}(x)\right|^{p(x)} d x \geqslant \int_{\Omega} w_{k}(x) \nabla u_{k}(x) d x-\int_{\Omega} \frac{1}{(a(x))^{q(x) / p(x)}} \frac{1}{q(x)}\left|w_{k}(x)\right|^{q(x)} d x
$$

and 


$$
\int_{\Omega} \frac{b(x)}{m(x)}\left|\nabla v_{k}(x)\right|^{m(x)} d x \geqslant \int_{\Omega} z_{k}(x) \nabla v_{k}(x) d x-\int_{\Omega} \frac{1}{(b(x))^{n(x) / m(x)}} \frac{1}{n(x)}\left|z_{k}(x)\right|^{n(x)} d x
$$

and by a direct calculation we get $J_{k}\left(u_{k}, v_{k}\right) \geqslant J_{k}^{D}\left(w_{k}, z_{k}\right)$. By (3.4), it follows that $J_{k}\left(u_{k}, v_{k}\right) \leqslant \inf _{(w, z) \in X_{k}^{d}} J_{k}^{D}(w, z) \leqslant J_{k}^{D}\left(w_{k}, z_{k}\right)$. Hence $J_{k}\left(u_{k}, v_{k}\right)=J_{k}^{D}\left(w_{k}, z_{k}\right)$ and by a direct calculation we have actually equalities in (3.10). Therefore by the properties of the Fenchel-Young transformation (3.2) holds. Assertion (3.3) follows by (3.4) and since $J_{k}^{D}\left(w_{k}, z_{k}\right)=J_{k}\left(u_{k}, v_{k}\right)$.

\section{Stability of solutions}

Now we take up the stability problem. We assume F1 - F3 and

F4: $F_{u}^{k}$ is differentiable in $u$ on $\left[-d_{0}, d_{0}\right]$ and in $v$ on $\left[-c_{0}, c_{0}\right]$ for almost all $x \in \Omega, F_{v}^{k}$ is differentiable in $u$ on $\left[-d_{0}, d_{0}\right]$ and in $v$ on $\left[-c_{0}, c_{0}\right]$ for almost all $x \in \Omega$. There exist constants $\beta_{1}, \beta_{2}, \beta_{3}, \beta_{4}>0$ (independent of $k$ ) such that

$$
\begin{aligned}
& \max _{u \in\left[-d_{0}, d_{0}\right]} \max _{v \in\left[-c_{0}, c_{0}\right]}\left|F_{u u}^{k}(x, u, v)\right| \leqslant \beta_{1}, \\
& \max _{u \in\left[-d_{0}, d_{0}\right]} \max _{v \in\left[-c_{0}, c_{0}\right]}\left|F_{v v}^{k}(x, u, v)\right| \leqslant \beta_{2}, \\
& \max _{u \in\left[-d_{0}, d_{0}\right]} \max _{v \in\left[-c_{0}, c_{0}\right]}\left|F_{u v}^{k}(x, u, v)\right| \leqslant \beta_{3}, \\
& \max _{u \in\left[-d_{0}, d_{0}\right]} \max _{v \in\left[-c_{0}, c_{0}\right]}\left|F_{v u}^{k}(x, u, v)\right| \leqslant \beta_{4} .
\end{aligned}
$$

Theorem 4.1. Assume F1, F2, F3, F4 and that for all $(u, v) \in X_{0}$ there exists a subsequence $\left\{k_{i}\right\}_{i=1}^{\infty}$ such that

$$
\lim _{i \rightarrow \infty} F_{u}^{k_{i}}(x, u(x), v(x))=F_{u}^{0}(x, u(x), v(x))
$$

and

$$
\lim _{i \rightarrow \infty} F_{v}^{k_{i}}(x, u(x), v(x))=F_{v}^{0}(x, u(x), v(x))
$$

almost everywhere in $\Omega$. For each $k=0,1,2, \ldots$ there exists a solution $\left(u_{k}, v_{k}\right)$ to the problem (1.2). There exists a subsequence $\left\{\left(u_{k_{n}}, v_{k_{n}}\right)\right\}_{n=1}^{\infty}$ of the sequence $\left\{\left(u_{k}, v_{k}\right)\right\}_{k=1}^{\infty}$ and $(\bar{u}, \bar{v}) \in X_{0}$ such that

$$
\left(u_{k_{n}}, v_{k_{n}}\right) \rightarrow(\bar{u}, \bar{v}) \in X_{0}, \text { weakly in } W_{0}^{1, p(x)}(\Omega) \times W_{0}^{1, m(x)}(\Omega)
$$

and

$$
\begin{aligned}
&-\operatorname{div}\left(a(x)|\nabla \bar{u}(x)|^{p(x)-2} \nabla \bar{u}(x)\right)=F_{u}^{0}(x, \bar{u}(x), \bar{v}(x)), \\
&-\operatorname{div}\left(b(x)|\nabla \bar{v}(x)|^{m(x)-2} \nabla \bar{v}(x)\right)=F_{v}^{0}(x, \bar{u}(x), \bar{v}(x)), \\
& \bar{u}(x) \operatorname{lon}=0, \quad \bar{v}(x) \text { lon }=0 .
\end{aligned}
$$


Proof: By Theorem 3.1 it follows that for each $k=0,1,2,3, \ldots$ there exists $\left(u_{k}, v_{k}\right) \in X_{k}$ satisfying (1.2). Since $X_{k} \subset X_{0}$ it follows that we may choose a weakly convergent subsequence in $W_{0}^{1, p(x)}(\Omega) \times W_{0}^{1, m(x)}(\Omega)$ which up to a subsequence may be assumed to be strongly convergent in $L^{p(x)}(\Omega) \times L^{m(x)}(\Omega)$ and convergent almost everywhere to $(\bar{u}, \bar{v})$. Due to $\mathrm{F} 1$

$$
\begin{aligned}
& \underset{x \in \Omega}{\operatorname{ess~sup}}\left|F_{u}^{k}(x, \bar{u}(x), \bar{v}(x))-F_{u}^{0}(x, \bar{u}(x), \bar{v}(x))\right| \leqslant \frac{2 a_{0} d_{0}}{C_{S}^{1} C_{1}^{p} C_{2}^{p}} \\
& \underset{x \in \Omega}{\operatorname{ess~sup}}\left|F_{v}^{k}(x, \bar{u}(x), \bar{v}(x))-F_{v}^{0}(x, \bar{u}(x), \bar{v}(x))\right| \leqslant \frac{2 b_{0} c_{0}}{C_{S}^{2} C_{1}^{m} C_{2}^{m}}
\end{aligned}
$$

By (4.3) and definition of $X_{k}$ we obtain that $\left\{-\operatorname{div}\left(a(\cdot)\left|\nabla u_{k}(\cdot)\right|^{p(x)-2} \nabla u_{k}(\cdot)\right)\right\}_{k=1}^{\infty}$ and $\left\{-\operatorname{div}\left(b(\cdot)\left|\nabla v_{k}(\cdot)\right|^{m(x)-2} \nabla v_{k}(\cdot)\right)\right\}_{k=1}^{\infty}$ are weakly convergent in $L^{q(x)}(\Omega)$ and $L^{n(x)}(\Omega)$, respectively to functions $d_{1} \in L^{q(x)}(\Omega), d_{2} \in L^{n(x)}(\Omega)$. We obtain that

$$
\begin{aligned}
0 \leqslant \int_{\Omega}\left\langle-\operatorname{div}\left(a(x)\left|\nabla u_{k}\right|^{p(x)-2} \nabla u_{k}\right)-\left(-\operatorname{div}\left(a(x)|\nabla u|^{p(x)-2} \nabla u\right)\right), u_{k}-u\right\rangle d x \\
\rightarrow \int_{\Omega}\left\langle d_{1}(x)-\left(-\operatorname{div}\left(a(x)|\nabla u|^{p(x)-2} \nabla u\right)\right), \bar{u}-u\right\rangle d x .
\end{aligned}
$$

Thus by the monotonicity of the $p(x)$-Laplacian we see that $d_{1}(x)=-\operatorname{div}\left(a(x)|\nabla \bar{u}|^{p(x)-2} \nabla \bar{u}\right)$ and similarly $d_{2}(x)=-\operatorname{div}\left(b(x)|\nabla \bar{v}|^{m(x)-2} \nabla \bar{v}\right)$.

We now prove that

$$
\begin{aligned}
& \lim _{i \rightarrow \infty} F_{u}^{k_{i}}\left(x, u_{k_{i}}(x), v_{k_{i}}(x)\right)=F_{u}^{0}(x, \bar{u}(x), \bar{v}(x)) \text { and } \\
& \lim _{i \rightarrow \infty} F_{v}^{k_{i}}\left(x, u_{k_{i}}(x), v_{k_{i}}(x)\right)=F_{v}^{0}(x, \bar{u}(x), \bar{v}(x)) \text { almost everywhere. }
\end{aligned}
$$

We show the first relation. We have

$$
\begin{gathered}
F_{u}^{k_{i}}\left(x, u_{k_{i}}(x), v_{k_{i}}(x)\right)-F_{u}^{0}(x, \bar{u}(x), \bar{v}(x))=F_{u}^{k_{i}}\left(x, u_{k_{i}}(x), v_{k_{i}}(x)\right) \\
-F_{u}^{k_{i}}(x, \bar{u}(x), \bar{v}(x))+F_{u}^{k_{i}}(x, \bar{u}(x), \bar{v}(x))-F_{u}^{0}(x, \bar{u}(x), \bar{v}(x))
\end{gathered}
$$

By the mean value theorem we observe that

$$
\begin{array}{r}
\left|F_{u}^{k_{i}}\left(x, u_{k_{i}}, v_{k_{i}}\right)-F_{u}^{k_{i}}(x, \bar{u}, \bar{v})\right| \leqslant \sup _{x \in \Omega} \sup _{(u, v) \in X_{0}} \sqrt{\left|F_{u u}^{k_{i}}\left(x, u_{k_{i}}, v_{k_{i}}\right)\right|^{2}+\left|F_{u v}^{k_{i}}\left(x, u_{k_{i}}, v_{k_{i}}\right)\right|^{2}} \\
\cdot \sqrt{\left|u_{k_{i}}-\bar{u}\right|^{2}+\left|v_{k_{i}}-\bar{v}\right|^{2}}
\end{array}
$$

Since $\left\{u_{k_{n}}\right\}_{n=1}^{\infty}$ and $\left\{v_{k_{n}}\right\}_{n=1}^{\infty}$ are convergent almost everywhere, by F4 it follows that

$$
\lim _{i \rightarrow \infty}\left(F_{u}^{k_{i}}\left(x, u_{k_{i}}(x), v_{k_{i}}(x)\right)-F_{u}^{k_{i}}(x, \bar{u}(x), \bar{v}(x))\right)=0 \text { almost everywhere }
$$


Thus from (4.6) using the above and by the assumption $\lim _{i \rightarrow \infty} F_{u}^{k_{i}}(x, \bar{u}(x), \bar{v}(x))$ $=F_{u}^{0}(x, \bar{u}(x), \bar{v}(x))$ we obtain (4.4). Since the weak limit is equal to an almost everywhere limit we'get (4.3).

By Theorem 3.1 there exists $\left(u_{0}, v_{0}\right) \in X_{0}$ such that $\inf _{(u, v) \in X_{0}} J_{0}(u, v)=J_{0}\left(u_{0}, v_{0}\right)$. The following corollary shows that under some additional assumptions $(\bar{u}, \bar{v})$ minimises $J_{0}$ on $X_{0}$.

Corollary 4.2. Under the assumptions of Theorem 4.1 if

$$
\limsup _{k \rightarrow \infty}\left(\int_{\Omega} F^{k}\left(x, u_{0}, v_{0}\right) d x-\int_{\Omega} F^{0}\left(x, u_{0}, v_{0}\right) d x\right) \leqslant 0
$$

and $\lim _{k \rightarrow \infty} F^{k}(x, \bar{u}, \bar{v})=F^{0}(x, \bar{u}, \bar{v})$ for almost everywhere $x \in \Omega$, then $(\bar{u}, \bar{v})$ minimises $J_{0}$ on $X_{0}$.

Proof: Let us suppose that $(\bar{u}, \bar{v})$ does not minimise $J_{0}$ on $X_{0}$, that is,

$$
J_{0}(\bar{u}, \bar{v})-J_{0}\left(u_{0}, v_{0}\right)>0
$$

where $\left(u_{0}, v_{0}\right)$ is a point minimising $J_{0}$ on $X_{0}$, provided by Theorem 3.1. Due to the weak lower semicontinuity of $J_{0}$ we have

$$
\liminf _{k \rightarrow \infty}\left(J_{0}\left(u_{k}, v_{k}\right)-J_{0}(\bar{u}, \bar{v})\right) \geqslant 0 .
$$

Hence, by

$$
\begin{aligned}
0<J_{0}(\bar{u}, \bar{v})-J_{0}\left(u_{0}, v_{0}\right)= & \left(J_{k}\left(u_{k}, v_{k}\right)-J_{0}\left(u_{0}, v_{0}\right)\right) \\
& -\left(J_{k}\left(u_{k}, v_{k}\right)-J_{0}\left(u_{k}, v_{k}\right)\right)-\left(J_{0}\left(u_{k}, v_{k}\right)-J_{0}(\bar{u}, \bar{v})\right)
\end{aligned}
$$

and by (4.7) the proof will be finished by showing that

$$
\lim _{k \rightarrow \infty}\left(J_{k}\left(u_{k}, v_{k}\right)-J_{0}\left(u_{k}, v_{k}\right)\right)=0
$$

and

$$
\liminf _{k \rightarrow \infty}\left(J_{k}\left(u_{k}, v_{k}\right)-J_{0}\left(u_{0}, v_{0}\right)\right) \leqslant 0
$$

We get

$$
\lim _{k \rightarrow \infty}\left(J_{k}\left(u_{k}, v_{k}\right)-J_{0}\left(u_{k}, v_{k}\right)\right)=\lim _{k \rightarrow \infty}\left(\int_{\Omega} F^{0}\left(x, u_{k}, v_{k}\right) d x-\int_{\Omega} F^{k}\left(x, u_{k}, v_{k}\right) d x\right)
$$

Since

$$
\begin{aligned}
\left|F^{0}\left(x, u_{k}, v_{k}\right)-F^{k}\left(x, u_{k}, v_{k}\right)\right| & \leqslant\left|F^{0}\left(x, u_{k}, v_{k}\right)-F^{0}(x, \bar{u}, \bar{v})\right| \\
+ & \left|F^{k}(x, \bar{u}, \bar{v})-F^{0}(x, \bar{u}, \bar{v})\right|+\left|F^{k}\left(x, u_{k}, v_{k}\right)-F^{k}(x, \bar{u}, \bar{v})\right|
\end{aligned}
$$


we have by the mean value theorem and by $\mathrm{F} 1$

$$
\begin{aligned}
& \left|F^{0}\left(x, u_{k}, v_{k}\right)-F^{0}(x, \bar{u}, \bar{v})\right| \\
& \leqslant \sup _{x \in \Omega} \sup _{(u, v) \in X_{0}} \sqrt{\left|F_{u}^{0}(x, u, v)\right|^{2}+\left|F_{v}^{0}(x, u, v)\right|^{2}} \sqrt{\left|u_{k}-\bar{u}\right|^{2}+\left|v_{k}-\bar{v}\right|^{2}} \\
& \leqslant \sqrt{a_{0}^{2} d_{k}^{2}+b_{0}^{2} c_{k}^{2}} \sqrt{\left|u_{k}-\bar{u}\right|^{2}+\left|v_{k}-\bar{v}\right|^{2}} \rightarrow 0, \\
& \left|F^{k}\left(x, u_{k}, v_{k}\right)-F^{k}(x, \bar{u}, \bar{v})\right| \rightarrow 0 \text {. }
\end{aligned}
$$

Since $F^{k}(x, \bar{u}, \bar{v}) \rightarrow F^{0}(x, \bar{u}, \bar{v})$ we obtain

$$
\lim _{k \rightarrow \infty}\left(\int_{\Omega} F^{0}\left(x, u_{k}, v_{k}\right) d x-\int_{\Omega} F^{k}\left(x, u_{k}, v_{k}\right) d x\right)=0,
$$

so (4.8) is shown.

Now since $\left(u_{k}, v_{k}\right)$ minimises $J_{k}$ and by (4.6) we have

$$
\begin{aligned}
\liminf _{k \rightarrow \infty}\left(J_{k}\left(u_{k}, v_{k}\right)-J_{0}\left(u_{0}, v_{0}\right)\right) & \leqslant \liminf _{k \rightarrow \infty}\left(J_{k}\left(u_{0}, v_{0}\right)-J_{0}\left(u_{0}, v_{0}\right)\right) \\
= & \liminf _{k \rightarrow \infty}\left(\int_{\Omega} F^{0}\left(x, u_{0}, v_{0}\right) d x-\int_{\Omega} F^{k}\left(x, u_{0}, v_{0}\right) d x\right) \leqslant 0
\end{aligned}
$$

so (4.9) is proved.

Investigation of the proof of Theorem 4.1 shows that we may weaken a bit its assumptions. Precisely, instead of F4 we assume $F_{u}^{k}$ and $F_{v}^{k}$ have property as in (4.4). Thus we have the following corollary.

Corollary 4.3. Assume F1, F2, F3 and that for all $(u, v) \in X_{0}$ there exists a subsequence $\left\{k_{i}\right\}_{i=1}^{\infty}$ such that $\lim _{i \rightarrow \infty} F_{u}^{k_{i}}(x, u(x), v(x))=F_{u}^{0}(x, u(x), v(x))$ and $\lim _{i \rightarrow \infty} F_{v}^{k_{i}}(x, u(x), v(x))=F_{v}^{0}(x, u(x), v(x))$ almost everywhere in $\Omega$. For each $k=0,1,2, \ldots$ there exists a solution $\left(u_{k}, v_{k}\right)$ to the problem (1.2), subsequence $\left\{\left(u_{k_{n}}, v_{k_{n}}\right)\right\}_{n=1}^{\infty}$ of the sequence $\left\{\left(u_{k}, v_{k}\right)\right\}_{k=1}^{\infty}$ and $(\bar{u}, \bar{v}) \in X_{0}$ satisfying (4.3). We assume that

$$
\begin{aligned}
& \lim _{i \rightarrow \infty} F_{u}^{k_{i}}\left(x, u_{k_{i}}(x), v_{k_{i}}(x)\right)=F_{u}^{0}(x, \bar{u}(x), \bar{v}(x)), \\
& \lim _{i \rightarrow \infty} F_{v}^{k_{i}}\left(x, u_{k_{i}}(x), v_{k_{i}}(x)\right)=F_{v}^{0}(x, \bar{u}(x), \bar{v}(x)) \text { almost everywhere }
\end{aligned}
$$

Then $\left(u_{k_{n}}, v_{k_{n}}\right) \rightarrow(\bar{u}, \bar{v}) \in X_{0}$ weakly in $W_{0}^{1, p(x)}(\Omega) \times W_{0}^{1, m(x)}(\Omega)$. If additionally $\lim _{k \rightarrow \infty} F^{k}(x, \bar{u}, \bar{v})=F^{0}(x, \bar{u}, \bar{v})$ for almost all $x \in \Omega$ and (4.6) holds, then $\inf _{(u, v) \in X_{0}} J_{0}(u, v)=$ $J_{0}(\bar{u}, \bar{v})$. 


\section{Continuous DEPENDENCE ON PARAMETERS}

Now we prove that system (5.1) depends continuously on a functional parameter $g_{k}$. We are interested in giving conditions asserting that if only $g_{k} \rightarrow \bar{g}$ in $L^{q(x)}(\Omega)$, then solutions $\left(u_{k}, v_{k}\right)$ to

$$
\begin{aligned}
&-\operatorname{div}\left(a(x)|\nabla u(x)|^{p(x)-2} \nabla u(x)\right)=F_{u}\left(x, u(x), v(x), g_{k}(x)\right), \\
&-\operatorname{div}\left(b(x)|\nabla v(x)|^{m(x)-2} \nabla v(x)\right)=F_{v}\left(x, u(x), v(x), g_{k}(x)\right), \\
&\left.u(x)\right|_{\partial \Omega}=0, u \in W_{0}^{1, p(x)}(\Omega),\left.\quad v(x)\right|_{\partial \Omega}=0, v \in W_{0}^{1, m(x)}(\Omega)
\end{aligned}
$$

converge (up to a subsequence) to the solution $(\bar{u}, \bar{v})$ to

$$
\begin{aligned}
& -\operatorname{div}\left(a(x)|\nabla u(x)|^{p(x)-2} \nabla u(x)\right)=F_{u}(x, u(x), v(x), \bar{g}(x)), \\
& -\operatorname{div}\left(b(x)|\nabla v(x)|^{m(x)-2} \nabla v(x)\right)=F_{v}(x, u(x), v(x), \bar{g}(x))
\end{aligned}
$$

Let $g_{k}$ and $\bar{g}$ be functional parameters taken from the set

$$
\left\{g: \Omega \rightarrow R^{m}: g \text { is measurable, } g(x) \in M \text { almost everywhere }\right\},
$$

where $M$ is a bounded and compact subset of $R^{m}$. Existence of solutions to (5.1) for each $k=0,1, \ldots$ is guaranteed by Theorem 3.1 .

We assume that for some $d>0, c>0$ we have

F5: $\left.\|1\|_{q(x)} \leqslant\left(1 / p^{-}\right)+1 / q^{-}\right)^{-1},\|1\|_{n(x)} \leqslant\left(1 / m^{-}+1 n^{-}\right)^{-1}$ and for all $g \in M$

$$
\begin{gathered}
C_{S}^{1} C_{1}^{p} C_{2}^{p} \text { ess sup } \max _{x \in \Omega} \max _{u \in[-d, d]}\left|F_{v \in[-c, c]}(x, u, v, g)\right| \leqslant a_{0} d, \\
C_{S}^{2} C_{1}^{m} C_{2}^{m} \text { ess sup } \max _{x \in \Omega} \max _{u \in[-d, d]} \mid F_{v}(x, u, c, c]
\end{gathered}
$$

F6: $F_{u}(x, 0,0,0) \neq 0, F_{v}(x, 0,0,0) \neq 0$ for almost all $x \in \Omega$, $x \mapsto F(x, 0,0,0)$ and $x \mapsto(F)^{*}(x, 0,0,0)$ are integrable on $\Omega$ for all $g \in M$.

F7: $F: \Omega \times[-d, d] \times[-c, c] \times M \rightarrow R$ is a Caratheodory function, that is, measurable in $x$ and continuous in $(u, v, g) . F$ is convex in $(u, v)$ on $[-d, d]$ $\times[-c, c]$ for almost all $x \in \Omega$ and all $g \in M$.

Since the notation changes, we now rewrite the definitions of $X_{k}$ and action functionals. We have for each $k=0,1,2, \ldots$ that $X_{k}=X$, where

$$
\begin{aligned}
X=\left\{(u, v) \in W_{0}^{1, p(x)}(\Omega) \times W_{0}^{1, m(x)}(\Omega),\|\nabla u\|_{p(x)}\right. & \\
& \left.\leqslant \frac{d}{C_{1}^{p} C_{2}^{p}}\|\nabla v\|_{m(x)} \leqslant \frac{c}{C_{1}^{m} C_{2}^{m}},|u(x)| \leqslant d,|v(x)| \leqslant c\right\}
\end{aligned}
$$


and

$$
\begin{aligned}
& J_{k}(u, v)=\int_{\Omega} \frac{a(x)}{p(x)}|\nabla u(x)|^{p(x)} d x+\int_{\Omega} \frac{b(x)}{m(x)}|\nabla v(x)|^{m(x)} d x-\int_{\Omega} F\left(x, u(x), v(x), g_{k}(x)\right) d x, \\
& J_{0}(u, v)=\int_{\Omega} \frac{a(x)}{p(x)}|\nabla u(x)|^{p(x)} d x+\int_{\Omega} \frac{b(x)}{m(x)}|\nabla v(x)|^{m(x)} d x-\int_{\Omega} F(x, u(x), v(x), \bar{g}(x)) d x .
\end{aligned}
$$

THEOREM 5.1. Assume F5-F7, $g_{k} \rightarrow \bar{g}$ in $L^{q(x)}(\Omega)$ and

$$
\limsup _{k \rightarrow \infty}\left(\int_{\Omega} F\left(x, u, v, g_{k}\right) d x-\int_{\Omega} F(x, u, v, \bar{g}) d x\right) \leqslant 0 .
$$

Then for each $k=0,1,2, \ldots$ there exists solutions $\left(u_{k}, v_{k}\right)$ to (5.1) minimising $J_{k}$ given by (5.2) on $X$. Moreover, up to a subsequence, $\left\{\left(u_{k}, v_{k}\right)\right\}$ converges in $L^{p(x)}(\Omega) \times L^{m(x)}(\Omega)$ to $(\bar{u}, \bar{v})$ being solution to

$$
\begin{aligned}
& -\operatorname{div}\left(a(x)|\nabla u(x)|^{p(x)-2} \nabla u(x)\right)=F_{u}(x, u(x), v(x), \bar{g}(x)), \\
& -\operatorname{div}\left(b(x)|\nabla v(x)|^{m(x)-2} \nabla v(x)\right)=F_{v}(x, u(x), v(x), \bar{g}(x))
\end{aligned}
$$

where $J_{0}(\bar{u}, \bar{v})=\inf _{(u, v) \in X} J_{0}(u, v)$.

Proof: We show that conditions of Corollary 4.3 are satisfied with $F^{k}(x, u, v)$ $:=F\left(x, u, v, g_{k}\right)$ and $F^{0}(x, u, v):=F(x, u, v, \vec{g})$. Let us fix $(u, v) \in X$. Clearly F5F7 imply F1-F3. By F5 it follows that $\left|F_{u}\left(x, u, v, g_{k}\right)\right|$ and $\left|F_{v}\left(x, u, v, g_{k}\right)\right|$ are bounded on $\Omega \times[-d, d] \times[-c, c] \times M$. By the generalised Krasnosielski Theorem [7] the Nemytskij operators

$$
\begin{aligned}
& L^{q(x)}(\Omega) \ni g \longmapsto F_{u}(\cdot, u(\cdot), v(\cdot), g(\cdot)) \\
& L^{q(x)}(\Omega) \ni g \longmapsto F_{v}(\cdot, u(\cdot), v(\cdot), g(\cdot))
\end{aligned}
$$

are well defined and continuous, that is,

$$
\begin{aligned}
& F_{u}\left(x, u(x), v(x), g_{k}(x)\right) \rightarrow F_{u}(x, u(x), v(x), \bar{g}(x)), \\
& F_{v}\left(x, u(x), v(x), g_{k}(x)\right) \rightarrow F_{v}(x, u(x), v(x), \bar{g}(x)) .
\end{aligned}
$$

Clearly (4.10) holds. Moreover, by F7 it follows that $F\left(x, u, v, g_{k}\right) \rightarrow F(x, u, v, \bar{g})$ almost everywhere in $\Omega$. Thus assertion follows by Corollary 4.3 .

Now we present a special form of system (5.1); that is, a special form of nonlinearities in which parameters are given linearly but the sequence of parameters is only weakly convergent. We provide also Theorem similar to Theorem 5.1.

5.1. LINEAR CASE. We assume that right hand side is in the form

$$
F\left(x, u, v, g_{k}\right)=F^{1}(x, u, v) g_{k}+F^{2}(x, u, v)
$$


ThEOREM 5.2. Assume F5-F7, $g_{k} \rightarrow \bar{g}$ weakly in $L^{q(x)}(\Omega)$ and

$$
x \mapsto F_{u}^{1}(\cdot, u(\cdot), v(\cdot)), x \mapsto F_{v}^{1}(\cdot, u(\cdot), v(\cdot))
$$

are in $L^{p(x)}(\Omega)$. Then for each $k=0,1,2, \ldots$ there exists solution $\left(u_{k}, v_{k}\right)$ to (5.1) minimising $J_{k}$ on $X$. Moreover, up to a subsequence, $\left\{\left(u_{k}, v_{k}\right)\right\}$ converges in $L^{p(x)}(\Omega)$ $\times L^{m(x)}(\Omega)$ to $(\bar{u}, \bar{v})$ being the solution to

$$
\begin{aligned}
& -\operatorname{div}\left(a(x)|\nabla u(x)|^{p(x)-2} \nabla u(x)\right)=F_{u}(x, u(x), v(x), \bar{g}(x)), \\
& -\operatorname{div}\left(b(x)|\nabla v(x)|^{m(x)-2} \nabla v(x)\right)=F_{v}(x, u(x), v(x), \bar{g}(x))
\end{aligned}
$$

and $J_{0}(\bar{u}, \bar{v})=\inf _{(u, v) \in X} J_{0}(u, v)$.

ProOF: We have since $\left\{g_{k}\right\}$ is weakly convergent and $F_{u}^{1}(\cdot, u(\cdot), v(\cdot)), F_{v}^{1}(\cdot, u(\cdot), v(\cdot))$ are in $L^{p(x)}(\Omega)$ that

$$
\begin{aligned}
& \int_{\Omega} F_{u}^{1}(x, u(x), v(x)) g_{k}(x) d x \rightarrow \int_{\Omega} F_{u}^{1}(x, u(x), v(x)) \bar{g}(x) d x \\
& \int_{\Omega} F_{v}^{1}(x, u(x), v(x)) g_{k}(x) d x \rightarrow \int_{\Omega} F_{v}^{1}(x, u(x), v(x)) \bar{g}(x) d x
\end{aligned}
$$

so

$$
\begin{aligned}
& \int_{\Omega} F_{u}(x, u(x), v(x)) g_{k}(x) d x \rightarrow \int_{\Omega} F_{u}(x, u(x), v(x)) \bar{g}(x) d x \\
& \int_{\Omega} F_{v}(x, u(x), v(x)) g_{k}(x) d x \rightarrow \int_{\Omega} F_{v}(x, u(x), v(x)) \bar{g}(x) d x
\end{aligned}
$$

The assertion follows by By Corollary 4.3.

\section{EXAMPLES}

We give now two examples of nonlinearities satisfying our growth assumptions.

Example 1. Let us first take

$$
F^{k}(x, u, v)=\left(C_{S}^{1} C_{1}^{p} C_{2}^{p}\right)^{-1}\left(e^{u}+f_{k}(x) u+\frac{1}{2} u^{2}\right)+\left(C_{S}^{2} C_{1}^{m} C_{2}^{m}\right)^{-1}\left(\frac{1}{2} e^{v}+\frac{1}{12} v^{4}-f_{k}(x) v\right)
$$

We assume that $\Omega$ is a bounded subset of $R^{N}$ and

$\mathrm{Z} 1 \quad a(x) \geqslant a_{0} \geqslant 2 \sqrt{e}+3, b(x) \geqslant b_{0} \geqslant \sqrt{3}+25 / 12$ for all $x \in \Omega$.

Z2 $f_{k} \in L^{1}(\Omega)$, ess sup $\left|f_{k}(x)\right|=1$ and meas $\left\{x \in \Omega \mid f_{k}(x)=-1 \vee f_{k}(x)\right.$

$$
=-1 / 2\}=0 \text {. }
$$


Clearly assumptions F2 and F3 are satisfied. $F_{u}^{k}, F_{v}^{k}$ are integrable respectively in $u, v$, on every compact subset of $R$. To conclude that F4 holds we only need to show (4.2). By Z2 and since $0<d_{k} \leqslant d_{0}, 0<c_{k} \leqslant c_{0}$ we get

$$
\begin{aligned}
& \max _{u \in\left[-d_{k}, d_{k}\right]} \max _{v \in\left[-c_{k}, c_{k}\right]}\left|F_{u u}^{k}(x, u, v)\right| \leqslant\left(C_{S}^{1} C_{1}^{p} C_{2}^{p}\right)^{-1}\left(e^{d_{0}}+1\right), \\
& \max _{u \in\left[-d_{k}, d_{k}\right]} \max _{v \in\left[-c_{k}, c_{k}\right]}\left|F_{v v}^{k}(x, u, v)\right| \leqslant\left(C_{S}^{2} C_{1}^{m} C_{2}^{m}\right)^{-1}\left(\frac{1}{2} e^{c_{0}}+c_{0}^{2}\right)
\end{aligned}
$$

We demonstrate that relations (2.4) hold. We obtain

$$
\begin{gathered}
C_{S}^{1} C_{1}^{p} C_{2}^{p} \operatorname{ess~sup}_{x \in \Omega} \max _{u \in\left[-d_{k}, d_{k}\right]} \max _{v \in\left[-c_{k}, c_{k}\right]}\left|F_{u}^{k}(x, u, v)\right| \leqslant e^{d_{k}}+d_{k}+1, \\
C_{S}^{2} C_{1}^{m} C_{2}^{m} \text { ess sup } \max _{x \in \Omega} \max _{u \in\left[-d_{k}, d_{k}\right]}\left|F_{v \in\left[-c_{k}, c_{k}\right]}^{k}(x, u, v)\right| \leqslant \frac{1}{2} e^{c_{k}}+\frac{1}{3} c_{k}^{3}+1 .
\end{gathered}
$$

By 21 the functions $x \mapsto e^{x}+\left(1-a_{0}\right) x+1$ and $x \mapsto(1 / 2) e^{x}+(1 / 3) x^{3}-b_{0} x+1$ are both nonpositive on the intervals $[1 / 2,5 / 2]$ and $[1 / 2,2]$, respectively. Thus

$$
\begin{aligned}
e^{d_{k}}+d_{k}+1 & \leqslant a_{0} d_{k}, \\
\frac{1}{2} e^{c_{k}}+\frac{1}{3} c_{k}^{3}+1 & \leqslant b_{0} c_{k},
\end{aligned}
$$

for $d_{k} \in[1 / 2,5 / 2]$ and $c_{k} \in[(1 / 2), 2]$ and we conclude that (2.4) holds. Therefore we may take any nondecreasing sequences $\left\{d_{k}\right\},\left\{c_{k}\right\}$ from $[1 / 2,5 / 2]$ and $[1 / 2,2]$, respectively and put $X_{k}$ as in (2.5).

As for stability we consider for $k=1,2, \ldots F^{k}$ as in the above but with $f_{k}$ which now reads

$$
f_{k}(x)=e^{-\left(k x^{2} / k+1\right)}
$$

Now F1, F2, F3, F4 and Z2 are obviously satisfied. Clearly (4.6) is also satisfied since $f_{k}(x) \rightarrow f_{0}(x):=e^{-x^{2}}$ uniformly on $\Omega$. By Corollary 4.3 it follows that from the sequence $\left\{\left(u_{k}, v_{k}\right)\right\}$ of solutions to

$$
\begin{aligned}
&-\operatorname{div}\left(a(x)|\nabla u(x)|^{p(x)-2} \nabla u(x)\right)=\left(C_{S}^{1} C_{1}^{p} C_{2}^{p}\right)^{-1}\left(e^{u}+u+e^{-\left(k x^{2} / k+1\right)}\right) \\
&-\operatorname{div}\left(b(x)|\nabla v(x)|^{m(x)-2} \nabla v(x)\right)=\left(C_{S}^{2} C_{1}^{m} C_{2}^{m}\right)^{-1}\left(\frac{1}{2} e^{v}+\frac{1}{3} v^{3}-e^{-\left(k x^{2} / k+1\right)}\right) \\
&\left.u(x)\right|_{\partial \Omega}=0, u \in W_{0}^{1, p(x)}(\Omega),\left.\quad v(x)\right|_{\partial \Omega}=0, v \in W_{0}^{1, m(x)}(\Omega)
\end{aligned}
$$

we may take subsequence converging to a certain $\left(u_{0}, v_{0}\right)$ being a solution to

$$
\begin{gathered}
-\operatorname{div}\left(a(x)|\nabla u(x)|^{p(x)-2} \nabla u(x)\right)=\left(C_{S}^{1} C_{1}^{p} C_{2}^{p}\right)^{-1}\left(e^{u}+u+e^{-x^{2}}\right), \\
-\operatorname{div}\left(b(x)|\nabla v(x)|^{m(x)-2} \nabla v(x)\right)=\left(C_{S}^{2} C_{1}^{m} C_{2}^{m}\right)^{-1}\left(\frac{1}{2} e^{v}+\frac{1}{3} v^{3}-e^{-x^{2}}\right), \\
\left.u(x)\right|_{\partial \Omega}=0, u \in W_{0}^{1, p(x)}(\Omega),\left.\quad v(x)\right|_{\partial \Omega}=0, v \in W_{0}^{1, m(x)}(\Omega) .
\end{gathered}
$$


Now we check that $F^{k}$ with $g_{k}(x):=f_{k}(x)=e^{-\left(k x^{2} / k+1\right)}$ satisfies assumptions of Theorem 5.2. We may rewrite it as follows

$$
\begin{aligned}
F^{k}(x, u, v)=F\left(x, u, v, g_{k}\right) & =\left(\left(C_{S}^{1} C_{1}^{p} C_{2}^{p}\right)^{-1} u+\left(C_{S}^{2} C_{1}^{m} C_{2}^{m}\right)^{-1} v\right) g_{k} \\
& +\left(C_{S}^{1} C_{1}^{p} C_{2}^{p}\right)^{-1}\left(e^{u}+\frac{1}{2} u^{2}\right)+\left(C_{S}^{2} C_{1}^{m} C_{2}^{m}\right)^{-1}\left(\frac{1}{2} e^{v}+\frac{1}{12} v^{4}\right)
\end{aligned}
$$

so it is in the form required in Theorem 5.2. It is clear that $g_{k} \rightarrow \bar{g}$ in $L^{q(x)}(\Omega)$ and that F5-F7 hold. Obviously the functions $x \mapsto F_{u}^{1}(\cdot, u(\cdot), v(\cdot)), x \mapsto F_{v}^{1}(\cdot, u(\cdot), v(\cdot)) \in$ $L^{p(x)}(\Omega)$. Thus assertion of Theorem 5.2 holds.

EXAMPLE 2. We consider

$$
\begin{aligned}
&-\operatorname{div}\left(a(x)|\nabla u(x)|^{p(x)-2} \nabla u(x)\right)=|x|^{2} \cdot|u(x)|^{\alpha_{k}(x)-1} \cdot u(x) \cdot v^{2}(x) \\
&+\frac{2}{\beta_{k}(x)+1} \cdot|x| \cdot|v(x)|^{\beta_{k}(x)+1} \cdot u(x)+|x|,
\end{aligned}
$$

$$
\begin{aligned}
&-\operatorname{div}\left(b(x)|\nabla v(x)|^{m(x)-2} \nabla v(x)\right)= \frac{2}{\alpha_{k}(x)+1} \cdot|x|^{2} \cdot|u(x)|^{\alpha_{k}(x)+1} \cdot v(x) \\
&+|x| \cdot|v(x)|^{\beta_{k}(x)-1} \cdot v(x) \cdot u^{2}(x)+|x|, \\
&\left.u(x)\right|_{\partial \Omega}=0, u \in W_{0}^{1, p(x)}(\Omega),\left.\quad v(x)\right|_{\partial \Omega}=0, v \in W_{0}^{1, m(x)}(\Omega) .
\end{aligned}
$$

Here $\Omega=B(0, \delta)$ is a ball in $R^{3}$ and for all $k=0,1, \ldots \alpha_{k}^{+}, \alpha_{k}^{-}>p^{+}>3$; $\beta_{k}^{+}, \beta_{k}^{-}>m^{+}>3$, where $\alpha_{k}^{+}=\sup _{x \in \Omega} \alpha_{k}(x), \alpha_{k}^{-}=\inf _{x \in \Omega} \alpha_{k}(x), \beta_{k}^{+}=\sup _{x \in \Omega} \beta_{k}(x)$, $\beta_{k}^{-}=\inf _{x \in \Omega} \beta_{k}(x)$. We also assume

W1 $a(x) \geqslant a_{0} \geqslant C_{S}^{1} C_{1}^{p} C_{2}^{p}, b(x) \geqslant b_{0} \geqslant C_{2}^{S} C_{1}^{m} C_{2}^{m}$ for all $x \in \Omega$.

W2 $\alpha_{k}^{+}=\beta_{k}^{+}$for all $k=1,2, \ldots$ and $\left\{\alpha_{k}^{+}\right\}$is bounded by $\bar{\alpha}^{+}$.

W3 $\delta$ satisfies the following inequalities

$$
\begin{aligned}
& 2^{\bar{\alpha}^{+}+2}\left(\delta^{2}+\frac{1}{2} \delta\right)+\delta \leqslant 1, \\
& 2^{\bar{\alpha}^{+}+2}\left(\frac{1}{2} \delta^{2}+\delta\right)+\delta \leqslant 1 .
\end{aligned}
$$

W4 $c_{k}=d_{k}=2-(1 / k)$ for all $k=1,2, \ldots$

Here

$F^{k}(x, u, v)=\frac{1}{\alpha_{k}(x)+1} \cdot|x|^{2} \cdot|u|^{\alpha_{k}(x)+1} \cdot v^{2}+\frac{1}{\beta_{k}(x)+1} \cdot|x| \cdot|v|^{\beta_{k}(x)+1} \cdot u^{2}+|x|(|u|+|v|)$

Obviously assumptions F2 and F3 are satisfied. We show now that (4.2) holds. Clearly $F_{u}^{k}$ is differentiable in $u$ on $\left[-d_{k}, d_{k}\right]$ and $F_{v}^{k}$ is differentiable in $v$ on $\left[-c_{k}, c_{k}\right]$ for almost all $x \in \Omega$. Moreover, for all $k$ by W2 and W4 we have

$$
\max _{u \in\left[-d_{k}, d_{k}\right]} \max _{v \in\left[-c_{k}, c_{k}\right]}\left|F_{u u}^{k}(x, u, v)\right|=\delta^{2} \alpha_{k}^{+} d_{k}^{a_{k}^{+}-1} c_{k}^{2}+\frac{2}{\beta_{k}^{-}+1} \delta c_{k}^{\beta_{k}^{+}+1} \leqslant 2^{\bar{\alpha}^{+}+1} \delta^{2} \bar{\alpha}^{+}+2^{\bar{\alpha}^{+}} \delta
$$


and similarly

$$
\max _{u \in\left[-d_{k}, d_{k}\right]} \max _{v \in\left[-c_{k}, c_{k}\right]}\left|F_{v v}^{k}(x, u, v)\right| \leqslant 2^{\bar{\alpha}^{+}} \delta^{2}+2^{\overline{\alpha^{+}+1}} \overline{\alpha^{+}} \delta
$$

Now we show that (2.4) is also satisfied. By W1 and W2 it is equivalent to showing that for all $k=1,2, \ldots$

$$
\begin{aligned}
& \delta^{2} \cdot\left(2-\frac{1}{k}\right)^{\alpha_{k}^{+}+2}+\frac{1}{2} \delta \cdot\left(2-\frac{1}{k}\right)^{\alpha_{k}^{+}+2}+\delta \leqslant 2-\frac{1}{k}, \\
& \frac{1}{2} \delta^{2} \cdot\left(2-\frac{1}{k}\right)^{\alpha_{k}^{+}+2}+\delta \cdot\left(2-\frac{1}{k}\right)^{\alpha_{k}^{+}+2}+\delta \leqslant 2-\frac{1}{k}
\end{aligned}
$$

or

$$
\begin{aligned}
& \left(2-\frac{1}{k}\right)^{\alpha_{k}^{+}+2}\left(\delta^{2}+\frac{1}{2} \delta\right) \leqslant 2-\frac{1}{k}-\delta, \\
& \left(2-\frac{1}{k}\right)^{\alpha_{k}^{+}+2}\left(\frac{1}{2} \delta^{2}+\delta\right) \leqslant 2-\frac{1}{k}-\delta
\end{aligned}
$$

which is true by W2 and W3. Thus we may put $X_{k}$ as in (2.5) with $c_{k}, d_{k}$ as in W4. Again we may conclude that from the sequence $\left\{\left(u_{k}, v_{k}\right)\right\}$ of solutions to (6.1) we may choose a subsequence converging to the solution $\left(u_{0}, v_{0}\right) \in X_{0}$ to

$$
\begin{aligned}
&-\operatorname{div}\left(a(x)|\nabla u(x)|^{p(x)-2} \nabla u(x)\right)=|x|^{2} \cdot|u(x)|^{\alpha_{0}(x)-1} \cdot u(x) \cdot v^{2}(x) \\
&+\frac{2}{\beta_{0}(x)+1} \cdot|x| \cdot|v(x)|^{\beta_{0}(x)+1} \cdot u(x)+|x|, \\
&-\operatorname{div}\left(b(x)|\nabla v(x)|^{m(x)-2} \nabla v(x)\right)= \frac{2}{\alpha_{0}(x)+1} \cdot|x|^{2} \cdot|u(x)|^{\alpha_{0}(x)+1} \cdot v(x) \\
&+|x| \cdot|v(x)|^{\beta_{0}(x)-1} \cdot v(x) \cdot u^{2}(x)+|x| .
\end{aligned}
$$

\section{REFERENCES}

[1] R.A. Adams, Sobolev spaces (Academic Press, New York, 1975).

[2] I. Ekeland and R. Temam, Convex analysis and variational problems (North-Holland, Amsterdam, 1976).

[3] X.L. Fan and D. Zhao, 'Sobolev embedding theorems for spaces $W^{k, p(x)}(\Omega)^{\prime}$, J. Math. Anal. App. 262 (2001), 749-760.

[4] X.L. Fan and H. Zhang, 'Existence of solutions for $p(x)$-Laplacian dirichlet problem', Nonlin. Anal. 52 (2003), 1843-1852.

[5] X.L. Fan and D. Zhao, 'On the spaces $L^{p(x)}(\Omega)$ and $W^{k, p(x)}(\Omega)$ ', J. Math. Anal. App. 263 (2001), 424-446.

[6] M. Galewski, 'New variational method for $p(x)$ - Laplacian equation', Bull. Austral. Math. Soc. 72 (2005), 53-65.

[7] M. Galewski, 'On the continuity of the Nemytskij operator between the spaces $L^{p_{1}(x)}$ and $L^{p_{2}(x)}$, Georgian Math. J. 13 (2006), 261-265. 
[8] M. Galewski and M. Plócienniczak, 'On the nonlinear Dirichlet problem with p(x)-Laplacian', Bull. Austral. Math. Soc. 75 (2007), 381-395.

[9] A. El Hamidi, 'Existence results to elliptic systems with nonstandart growth conditions', J. Math. Anal. Appl. 300 (2004), 30-42.

[10] D. Idczak, 'Stability in semilinear problems', J. Differential Equations 162 (2000), 64-90.

[11] M. Ruzicka, Electrorheological fluids: Modelling and mathematical theory, Lecture Notes in Mathematics 1748 (Springer-Verlag, Berlin, 2000).

[12] S. Walczak, 'On the continuous dependence on parameters of solutions of the Dirichlet problem. Part I. Coercive Case, Part II. The Case of Saddle Points', Acad. Roy. Belg. Bull. Cl. Sci. 6 (1995), 247-273.

[13] S. Walczak, 'Continuous dependence on parameters and boundary data for nonlinear P.D.E. coercive case', Differential Integral Equations 11 (1998), 35-46.

[14] V.V. Zhikov, 'Averaging of functionals of the calculus of variations and elasticity theory', Math. USSR Izv. 29 (1987), 33-66.

Faculty of Mathematics and Computer Science

University of Lódź

Banacha 22

90-238 Lódź

Poland

e-mail: galewski@math.uni.lodz.pl

plo@math.uni.lodz.pl 